\title{
Lois et règlements sur la faune sauvage à Mada- gascar : Progrès accomplis et besoins du futur
}

Andrinajoro R. Rakotoarivelo', Julie H. Razafimanahaka', Sahondra Rabesihanaka", Julia P. G. Jones"' and Richard K. B. Jenkins ${ }^{1,11, \text { IV }}$

\section{RÉSUMÉ}

Les lois et règlements déterminant les niveaux de protection des espèces de la faune et de la flore sauvages sont des indicateurs importants de l'importance qu'accorde un pays à la conservation de sa biodiversité. Dans cette revue, nous évaluons la cohérence entre les lois et règlements portant sur la gestion de la faune sauvage à Madagascar, en considérant la législation nationale, les conventions internationales ratifiées et la Liste Rouge de I'UICN pour les confronter aux réalités locales. Suite à nos analyses, nous pouvons conclure que Madagascar dispose d'un cadre juridique adéquat pour réglementer la protection et I'exploitation des animaux sauvages. Cependant, des révisions et mises à jour sont nécessaires, particulièrement en ce qui concerne la liste des espèces dans les différentes catégories et la facilitation de la mise en application de la loi.

\section{ABSTRACT}

In many countries wildlife species are threatened by hunting for meat or collection for the pet trade. Wildlife laws which control where these activities can occur, limit the timing of exploitation, or provide strict protection for some species are therefore an important component of the conservation strategy. However it is important that these wildlife laws reflect the ecology and threat status of the species concerned, and that they are aligned with any relevant international conventions. In this article we discuss the legal framework for exploiting and protecting tetrapod species in Madagascar. We review the 2006 update to wildlife legislation with respect to international treaties, other national legislation and the IUCN Red List of Threatened Species. We also present a summary of the different categories of hunting (sport, commercial, scientific, and subsistence) and the control of hunting in protected areas. Madagascar has a sound legal framework for the use and protection of wildlife and the classification of species into protected, pest and legally hunted is clear and mostly fits well with the species' classification according to the IUCN Red List and CITES. A revision of the protected species list managed is needed however to (i) include marine mammals that
\end{abstract}

\author{
Correspondence: \\ Andrinajoro Rakotoarivelo \\ Madagasikara Voakajy \\ B. P. 5181, Antananarivo, Madagascar \\ E-mail: andrinajoro@moov.mg
}

are protected by fisheries law and the Convention on Migratory Species and to (ii) better reflect the rights of people whose livelihoods rely heavily on the income or protein derived from hunting animals. Renewed effort to communicate and enforce wildlife legislation is needed, especially regarding the illegal hunting and export of protected species. This would also support the ongoing initiative to expand the protected area system and could be integrated into a revised National Biodiversity Strategy and Action Plan that Madagascar should produce for 2011-2020 as part of its commitment to implementing the Convention on Biological Diversity.

KEYWORDS: Conservation, exploitation, hunting, legislation, protected species.

MOTS CLEFS : Conservation, exploitation, chasse, législation, espèces protégées.

\section{INTRODUCTION}

La chasse pour la consommation et la domestication figure parmi les pressions directes qui menacent les vertébrés dans les pays tropicaux (Wilkie et al. 1992, Bakkar et al. 2001, Fa et al. 2002, Corlett 2007). Afin de minimiser les impacts négatifs, la mise en place de lois définissant des aires d'exclusion de chasse, interdisant l'exploitation cynégétique de certaines espèces ou limitant l'exploitation de gibiers à une période définie, fait partie des stratégies adoptées par plusieurs pays. Si dans la pratique, un système de législation clair et bien compris n'est pas suffisant pour assurer la conservation, il constitue une première étape essentielle (Keane et al. 2008). Dans les pays en voie de développement, I'insuffisance des ressources humaines et financières limite la capacité des gouvernements à mettre en application la législation sur la conservation des espèces (Wilkie et al. 1992, Peres et Terborgh 1995, Wilkie et al. 2001).

Madagascar est un pays de mégadiversité avec une nature d'une extrême richesse et un degré d'endémisme élevé. II fait partie des régions prioritaires en matière de conservation de la biodiversité (Myers et al. 2000). Au cours de ces dernières 


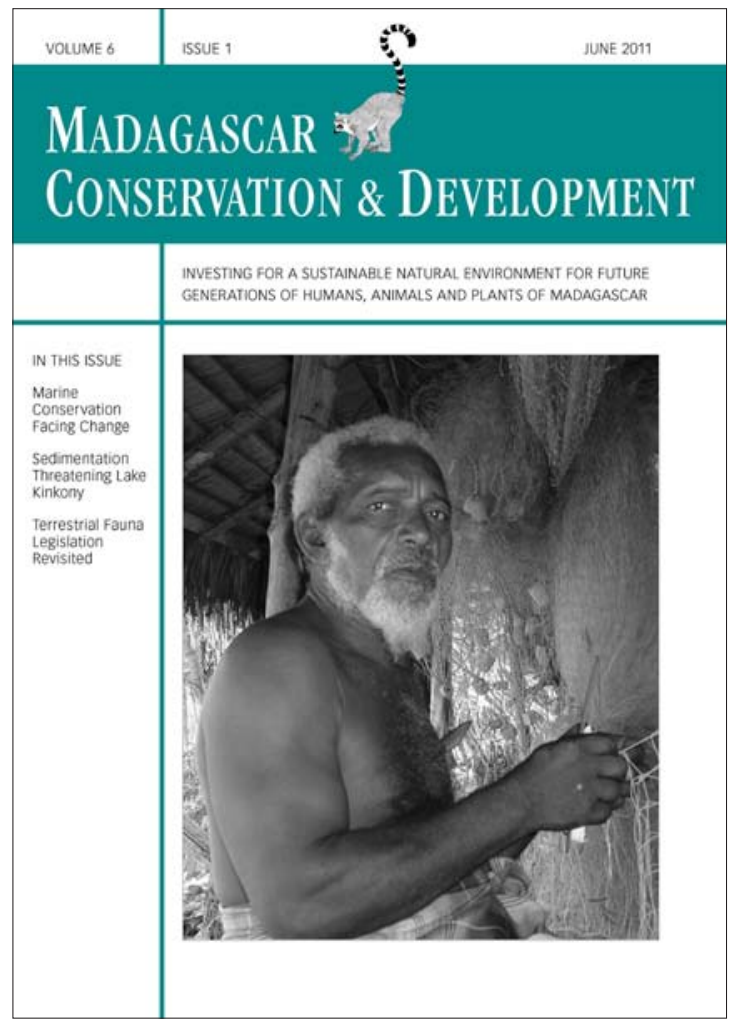

Madagascar Conservation \& Development is the journal of Indian Ocean e-Ink. It is produced under the responsibility of this institution. The views expressed in contributions to MCD are solely those of the authors and not those of the journal editors or the publisher.

All the Issues and articles are freely available at http://www.journalmcd.com

Contact Journal MCD

info@journalmcd.net for general inquiries regarding MCD funding@journalmcd.net to support the journal

Madagascar Conservation \& Development Institute and Museum of Anthropology University of Zurich

Winterthurerstrasse 190

CH-8057 Zurich, Switzerland

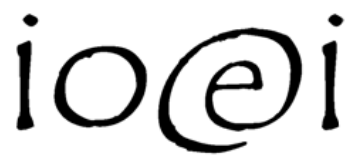

Indian Ocean e-Ink

Promoting African Publishing and Education www.ioeink.com

Missouri Botanical Garden (MBG)

Madagascar Research and Conservation Program BP 3391

Antananarivo, 101, Madagascar 
années, le niveau de connaissance de l'état de la biodiversité s'est développé, et une estimation récente montre que I'écosystème malgache abrite environ 12000 espèces de plantes, 363 espèces de reptiles, 238 espèces d'amphibiens, 283 espèces d'oiseaux, 165 espèces de poissons et 99 espèces de lémuriens (Goodman et Benstead 2005). Cependant, la chasse aux espèces de la faune sauvage est répandue sur l'ensemble de I'île (Goodman et Raselimanana 2003, Goodman et al. 2004, Rakotondravony 2006). Des études portant sur des ossements de lémuriens subfossiles ont montré que la chasse était déjà pratiquée il y a 2000 ans (Perez et al. 2005). De nos jours, la viande de brousse prend une place plus ou moins importante dans l'alimentation des populations rurales malgaches (Rakotondravony 2006). Les vertébrés concernés par la chasse sont les cétacés, les siréniens, les lémuriens, les tenrecs, les sangliers, les carnivores, les chauves-souris, les oiseaux, les reptiles et les amphibiens (Randriamanalina et al. 2000, Garcìa et Goodman 2003, Goodman et Raselimanana 2003, Nicoll 2003, Goodman et al. 2004, Rakotondravony 2006, Jenkins et Racey 2008, Kiszka et al. 2008, Golden 2009, Jenkins et al. 2009, Randrianandrianina et al. 2010). Alors que les volumes concernés par la chasse n'avaient été guère abordés dans I'histoire, la question a récemment reçu bien plus d'attention. Plus récemment encore, certains auteurs ont soupçonné que les collectes illégales d'espèces protégées pour la consommation locale et le commerce international ont augmenté depuis le début de la crise politique en mars 2009 (Barret et Ratsimbazafy 2009, Draper 2010).

Les premières actions visant à promulguer des lois destinées à protéger les animaux sauvages de Madagascar remontent à 1923 et portaient alors sur la tortue à écailles Eretmochelys imbricata et la tortue verte Chelonia mydas (Rakotonirina et Cooke 1994). En 1960, une ordonnance incluant un plus large éventail d'espèces a été promulguée (ordonnance 60-126 du 3 octobre 1960). Plusieurs autres lois et textes ont ultérieurement été adoptés au niveau national, et les règlementations nationales et les conventions internationales ont été appliquées parallèlement pour la gestion de l'exploitation et de l'aménagement des forêts, des aires protégées et des espèces terrestres et aquatiques.

En ne considérant que les espèces marines, cooke (sous presse) montre que la réglementation portant sur les espèces protégées à Madagascar présente plusieurs incohérences. Cette revue compile les lois et règlementations en vigueur pour régir la chasse et la collecte des espèces de faune sauvage à Madagascar. Nous comparons la catégorisation appliquée au niveau national avec celles des conventions et traités internationaux ratifiés par Madagascar (Convention sur la Diversité Biologique - CDB, Convention sur le Commerce International des Espèces de Faune et de Flore - CITES, Convention de Bonn - CMS, Convention d'Alger, Convention de Nairobi), les évaluations des scientifiques pour la Liste Rouge de I'UICN et les réalités locales. Si la présente revue est limitée aux tétrapodes, nous n'ignorons pas que les poissons et les invertébrés sont également exploités pour la consommation et la commercialisation à Madagascar.

\section{MÉTHODOLOGIE}

Cette étude a été basée sur deux méthodes principales que sont la documentation et l'interview. La documentation nous a permis de compiler toutes les lois et règlementations rela- tives à la chasse à Madagascar. Les documents ont été obtenus de trois directions sous le Ministère de I'Environnement et des Forêts (MEF), Direction Générale des Forêts (DGF) sises à Antananarivo: (i) la Direction de la Conservation de la Biodiversité et du Système des Aires Protégées (DCBSAP), (ii) la Direction de la Valorisation des Ressources Naturelles (DVRN) et (iii) la Direction Régionale de l'Environnement et des Forêts (DREF) Analamanga. D'autres documents proviennent du Ministère de la Pêche et des Ressources Halieutiques (MPRH) d'Antananarivo et une revue préparée par cooke (sous presse) a complété l'ensemble. Les informations sur les conventions internationales ainsi que les statuts de conservation de I'UICN ont été obtenues en ligne (wWw.iucnredlist.org, www.cites.org, www.cms.int, www.unep.org/Nairobiconvention, www.africa-union.org/root/au/Documents/Treaties). Des interviews ont été menées avec les responsables et les juristes affiliés au MEF afin de mieux cerner l'interprétation et les modalités d'application des textes en vigueur. Des expertises régionales et des documents n'existant que dans les autres régions nous ont certainement échappé mais le temps imparti à cette étude nous a limités aux enquêtes dans la région d'Antananarivo.

Une base de données comprenant 1137 espèces présentes à Madagascar a été établie à partir des informations disponibles sur la Liste Rouge de I'UICN et d'autres références bibliographiques pour les taxons non représentés sur la Liste Rouge (cas de Reptiles - Glaw et Vences 2007) ou d'espèces récemment découvertes (ex. Andriantompohavana et al. 2007, Craul et al. 2007, Goodman et al. 2009). Les catégories auxquelles chaque espèce appartient au niveau national, selon I'UICN et suivant les conventions internationales ont également été compilées dans la base de données.

\section{RÉSULTATS}

CONVENTIONS INTERNATIONALES. Madagascar a ratifié quatre conventions internationales liées à l'utilisation et la conservation des animaux. La Convention sur la Diversité Biologique (CDB) a été ratifiée le 4 mars 1996 (Ioi 1995-013 et décret 1995-695). Cette convention a pour objectif de conserver la diversité biologique par I'utilisation durable de ses éléments et une distribution équitable des bénéfices. Elle n'influe pas directement sur les modes d'exploitation ou de protection des espèces mais elle fournit un cadre adapté dans la Stratégie Nationale pour la Gestion de la Biodiversité. La Convention de Ramsar, ratifiée en 1998, encourage I'utilisation rationnelle des zones humides mais ne fournit pas d'informations au niveau des espèces (loi 1998-003 et décret 1998-261). La Convention sur le Commerce International des Espèces de Faune et de Flore Sauvages Menacées d'Extinction (CITES) porte exclusivement sur les espèces menacées par le commerce international mais ne contrôle pas les exploitations nationales pour la consommation des ménages ou d'autres utilisations. Elle a été ratifiée le 16 août 1975 (ordonnance 1975-014). La Convention sur les Espèces Migratrices (CMS) a été ratifiée le $1^{\text {er }}$ janvier 2007, elle a pour objectif de protéger les espèces migratrices sur l'ensemble de leur territoire. Pour les espèces inscrites à l'annexe 1 de cette convention, les parties signataires s'engagent à interdire les prélèvements dans la nature mais peuvent autoriser les collectes à des fins scientifiques ou I'utilisation traditionnelle pour la subsistance. Les espèces figurant à l'annexe 2 sont menacées dans une certaine mesure et leur survie dépend des 
accords internationaux pour leur conservation et leur gestion. Madagascar a signé l'Accord sur les tortues marines, le dugong et les oiseaux de proie ainsi que l'Accord sur la Conservation des Oiseaux d'eau Migrateurs d'Afrique-Eurasie.

CONVENTIONS AFRICAINES. La Convention de Nairobi

sur la protection, la gestion et le développement de I'environnement marin et côtier de la région de l'Afrique de I'Est a été signée à Nairobi en 1985 et ratifiée par Madagascar en 1998 (Ioi 1998-004 et décret 1998-260). Cette convention liste les espèces animales dans trois annexes et les parties signataires s'engagent à protéger les espèces listées. La Convention d'Alger (1968) a été ratifiée par Madagascar en 1970 (Loi 1970-004). Elle engage les parties à protéger toutes les espèces d'une classe A et permettre la chasse ou la collecte des espèces d'une classe B sous réserve d'une autorisation.

LÉGISLATION NATIONALE. Madagascar a adopté

différentes règlementations portant sur I'utilisation et la conservation des espèces animales sauvages, notamment à travers le Ministère de l'Environnement et des Forêts (MEF) et le Ministère de la Pêche et des Ressources Halieutiques (MPRH). Le décret du 23 mai 1923 pour la protection de deux espèces de tortues marines interdisait la capture des femelles en nidification et de tout individu dont la longueur de la carapace était inférieure à $50 \mathrm{~cm}$ (Rakotonirina et Cooke 1994). Ces textes ont été appuyés par celui du 23 octobre 1924 pour la protection des tortues marines en nidification (Rakotonirina et Cooke 1994). Les ordonnances 1960-126 et 1960-128 concernent I'exploitation (ex. chasse et pêche) et la protection des espèces et des habitats ; elles ont été complétées par l'arrêté 327-MAP/ FOR de 1961 portant sur les saisons de chasse et I'ordonnance 1962-20 sur la protection des lémuriens. La convention CITES a été ratifiée par Madagascar par I'ordonnance 1975-014 et mise en application par le décret 2005-848. Des révisions des lois sur la chasse, la convention CITES et la protection des espèces ont été effectuées en 2006 et mises en applications, par les décrets 2006-098 et 2006-400. Les lois et règlements portant sur la création et la gestion des aires protégées ont aussi des influences sur l'exploitation des animaux sauvages (décret 2005-848). La pêche et l'aquaculture ont toujours été gouvernées par un ministère distinct de celui en charge de I'environnement et des forêts, il s'agit actuellement du MPRH. Ce ministère régit I'exploitation de toutes les espèces aquatiques (ex. ordonnances 1985-013 et 1993-022), y compris les poissons et les invertébrés aquatiques (que nous ne traitons pas dans la présente revue) et est aussi responsable de contrôler la collecte de cétacés, de siréniens, de reptiles aquatiques et de certains amphibiens.

PROTECTION ET GESTION DES ESPÈCES. Le décret

2006-400 classifie les espèces de faune sauvage de Madagascar en trois catégories au niveau du MEF : les espèces protégées (catégorie 1) réparties en deux classes (1 et 2), les espèces nuisibles (catégorie 2) et les gibiers (catégorie 3). La liste associée à ce décret présente 654 vertébrés (Tableau 1), de sorte que 480 des espèces de notre base de données en sont absentes. Au niveau du MPRH, I'ordonnance 1993-022 stipule qu'il est interdit de capturer et de tuer tout mammifère marin y compris les baleines et les dauphins sur l'ensemble du territoire de Madagascar, de sorte que ces espèces sont protégées de facto.

Les espèces protégées de la catégorie 1 sont séparées en deux classes dont la première, catégorie 1, classe 1 (Cat. 1.1),
TABLEAU 1. Les 1134 espèces des classes des Mammifères, des Oiseaux des Reptiles et des Amphibiens dans les catégories de protection des espèces de la faune sauvage dans la législation sur la chasse malgache (annexe du décret 2006-400)

\begin{tabular}{|c|c|c|c|c|c|}
\hline \multirow[t]{2}{*}{ Classes } & \multicolumn{2}{|c|}{ Espèces protégées } & \multirow{2}{*}{$\begin{array}{l}\text { Nuisibles } \\
\text { Cat. } 2.0\end{array}$} & \multirow{2}{*}{$\begin{array}{l}\text { Gibier } \\
\text { Cat. } 3.0\end{array}$} & \multirow{2}{*}{$\begin{array}{l}\text { Non } \\
\text { catégorisées }\end{array}$} \\
\hline & Cat. 1.1 & Cat. 1.2 & & & \\
\hline Amphibia & 25 & 37 & 0 & 46 & 124 \\
\hline Aves & 62 & 66 & 2 & 127 & 37 \\
\hline Mammalia & 107 & 13 & 5 & 5 & 111 \\
\hline Reptilia & 26 & 114 & 0 & 19 & 208 \\
\hline Totaux & 220 & 230 & 7 & 197 & 480 \\
\hline
\end{tabular}

inclut les espèces qui bénéficient d'une protection absolue sur I'ensemble du territoire de la république. La chasse, la capture, la détention, la consommation et la commercialisation des espèces de la classe Cat. 1.1 sont strictement interdites sous toutes ses formes. Toutefois, il est prévu que la capture et l'exportation des espèces de cette classe dans le cadre d'études et d'échanges scientifiques, ainsi que leur reproduction en captivité ou leur exposition soient autorisées sous réserve d'une autorisation émanant du gouvernement. Au total, 220 espèces se trouvent dans cette catégorie dont $49 \%$ sont des mammifères, $28 \%$ des oiseaux, $12 \%$ des reptiles et $11 \%$ des amphibiens (Tableau 1).

Quarante-six pour cent des espèces dans la Cat. 1.1 sont des espèces menacées sur la Liste Rouge de I'UICN (En Danger Critique d'Extinction - CR, En Danger - EN ou Vulnérable - VU), $4 \%$ sont Quasi-Menacées (NT) et $16 \%$ de Préoccupation Mineure (LC) (Tableau 2). Selon la réglementation malgache, Iorsqu'une espèce est reconnue comme menacée dans son statut de conservation, elle bénéficie d'une protection totale, mais $24 \%$ des espèces En Danger Critique d'Extinction de Madagascar ne sont pas inclus dans la Cat. 1.1. Parmi elles, Cophyla berara (amphibien ne figurant sur aucune liste), Erymnochelys madagascariensis, Phelsuma antanosy et $P$. pronki (reptiles inscrits dans la Cat. 1.2 ci-dessous), Mantella aurantiaca et M. milotympanum (amphibiens inscrits dans la Cat.1.2 ci-dessous). Alors que $51 \%$ des espèces En Danger sont dans la Cat. 1.1, 41\% ne sont pas inscrites à l'annexe du décret 2006-400.

Cinq tortues marines et le Crabier blanc Ardeola idae figurent dans la Cat. 1.1 et dans I'annexe 1 (espèces protégées) de la CMS alors que six espèces de baleines inscrites à I'annexe 1 (espèces protégées) de la CMS ne sont pas listées dans l'annexe du décret 2006-400 (Matériel Supplémentaire). Seule une espèce de l'annexe 2 de la CMS figure dans la Cat. 1.1 alors que quatre espèces d'oiseaux pélagiques et le dugong sont menacés d'après la Liste Rouge de I'UICN et n'apparaissent que dans l'annexe 2 de la CMS.

TABLEAU 2. Nombre d'espèces dans les catégories des espèces de la faune sauvage de la législation nationale malgache en fonction de leur statut de conservation de la Liste Rouge de I'UICN (CR = En Danger Critique d'Extinction, EN = En Danger, VU = Vulnérable, NT = Quasi Menacé, LC = Préoccupation Mineure, DD = Données Insuffisantes)

\begin{tabular}{lcccccc}
\hline & \multicolumn{7}{c}{ Catégories UICN des espèces citées dans } \\
& CR & EN & VU & NT & LC & DD \\
\hline Espèces protégées Cat. 1.1 & 19 & 39 & 46 & 20 & 35 & 43 \\
\hline Espèces protégées Cat. 1.2 & 5 & 7 & 15 & 8 & 93 & 3 \\
\hline Nuisibles & 0 & 0 & 0 & 0 & 7 & 0 \\
\hline Gibier & 0 & 1 & 4 & 8 & 169 & 2 \\
\hline
\end{tabular}


Cent vingt-deux espèces de la Cat. 1.1 sont inscrites à I'annexe 1 de la CITES et 22 autres à I'annexe 2. Onze espèces dans l'annexe 1 de la CITES (huit baleines, le dugong et deux serpents) ne sont pas listées dans le décret 2006-400. Treize espèces dans I'annexe 2 de la Convention de Nairobi (espèces nécessitant une protection) sont dans la Cat.1.1 et huit sont dans I'annexe 4 (espèces migratrices protégées). Cent quatre espèces protégées dans la Convention d'Alger (classe A) sont aussi dans la Cat.1.1, mais le dugong n'est pas inclus dans la liste des espèces protégées de Madagascar.

La seconde classe, catégorie 1, classe 2, ou Cat. 1.2 regroupe les espèces qui peuvent être chassées ou capturées sous réserve de l'obtention d'une autorisation et dans le respect de quotas de collecte pour chaque espèce, fixés chaque année par l'organe de gestion de la CITES sur proposition de l'autorité scientifique. Au total, 230 espèces se trouvent dans cette classe incluant des mammifères $(6 \%)$ et des reptiles (Tableau 1 ). Par rapport aux catégories de la Liste Rouge de I'UICN, 12\% des espèces de cette catégorie sont menacées (Tableau 2). La majorité des espèces de la Cat.1.2 n'ont pas encore été évaluées par I'UICN (48\%) ou sont de Préoccupation Mineure (41\%), cinq espèces sont En Danger Critique d'Extinction et sept sont En Danger (Tableau 2).

Cinq oiseaux d'eau inscrits à l'annexe 2 de la CMS figurent aussi dans la Cat. 1.2. II y a une forte similarité entre la Cat. 1.2 et I'annexe 2 de la CITES, avec 131 espèces présentes sur les deux listes. Cependant, 104 autres espèces dans la Cat. 1.2 ne figurent pas dans la liste de la CITES et sont par conséquent collectées uniquement au niveau national, comprenant 72 espèces de Préoccupation Mineure. Certaines de ces espèces sont chassées pour la consommation (ex. Setifer setosus), d'autres sont récoltées pour le commerce international (Zonosaurus spp.). La Cat. 1.2 est similaire à la classe B de la Convention d'Alger bien que seules cinq espèces de la Cat. 1.2 figurent parmi les 36 espèces de la classe B. Lorsqu'une espèce protégée (Cat. 1.1 et 1.2) représente un danger ou cause des dommages aux personnes ou à leurs biens, I'administration forestière locale peut décider de son abattage ou son transfert dans une autre zone, à conditions que les faits aient été confirmés par les responsables administratifs locaux (maires, chefs de Fokontany).

Les espèces nuisibles (Cat. 2) sont des espèces dont la chasse, la capture et la consommation sont autorisées toute I'année. Onze espèces appartiennent à cette catégorie dont quatre espèces de mammifères et sept espèces d'oiseaux. Ces espèces sont généralement considérées comme des ravageurs des productions agricoles ou des prédateurs d'animaux domestiques. II s'agit d'espèces de Préoccupation Mineure selon les critères de I'UICN et elles n'apparaissent pas dans les annexes de la CITES ou de la CMS.

Les espèces de gibier (Cat. 3) sont des espèces qui peuvent être chassées ou capturées par des personnes titulaires d'une autorisation de chasse pendant la période d'ouverture de la chasse. Suivant l'arrêté 327-MAP/FOR du 6 février 1961, cette période est définie annuellement par le Ministère chargé de I'Administration de l'Environnement et des Forêts. Cette catégorie comprend 197 espèces, les oiseaux étant les plus nombreux (64\%). Par rapport à la Liste Rouge de I'UICN, cette catégorie est composée de 169 espèces de Préoccupation Mineure, une espèce En Danger, quatre espèces Vulnérables et huit espèces Quasi Menacées. Trois espèces d'oiseaux de la Cat. 3 figurent dans l'annexe 2 de la CMS et sept espèces figurent dans l'annexe 2 de la CITES, y compris la chauve-souris Pteropus rufus.

Rappelons que sont interdites, même en période d'ouverture de la chasse, les pratiques suivantes:

1. la poursuite, l'approche et le tir du gibier en véhicule ou bateau à moteur ;

2. la chasse aux phares, à la lanterne et en général à I'aide de tous engins éclairants ;

3. la chasse à l'arme de guerre ou à l'aide de projectiles explosifs ;

4. la chasse de nuit (entre le coucher et le lever du soleil);

5. la chasse à l'aide de drogue, appâts empoisonnés, filets, pièges et fosses ;

6. la chasse au moyen des feux ;

7. la chasse au moyen d'armes de fabrication locale (à I'exception du droit d'usage).

AUTORISATION DE CHASSE ET SON EXERCICE. Selon I'ordonnance 60-126 et le décret 61-093, il existe trois types d'autorisation de chasse dont la chasse sportive, la chasse à but scientifique et la chasse à but commercial. L'autorisation de chasse sportive donne droit au titulaire d'abattre, pendant la période d'ouverture de la chasse, les animaux classés comme " gibier » et comme "nuisibles » (Cat. 2 et Cat. 3). Rappelons que I'exercice de ce droit de chasser avec une arme à feu est subordonné à l'obtention d'une autorisation de détention d'armes qui est délivrée par le service responsable de la défense. L'autorisation de chasse sportive avec port d'arme est délivrée par la Direction Générale des Forêts ou les Directions Régionales de I'Environnement et des Forêts; elle est valable pour l'année calendaire au cours de laquelle elle a été délivrée et ouvre à une perception de redevances.

La chasse à but scientifique est une autorisation de collecte ou de chasse réalisée dans un objectif scientifique et peut porter sur des espèces protégées en soustrayant son détenteur aux interdictions prescrites par les articles 2, 4, 10 et 11 de I'ordonnance 60-126. Cette autorisation ne peut être délivrée que par la DGF à des personnes ou organismes en possession d'une autorisation de recherche qui stipule ce type de prélèvement. La Commission Ad hoc Faune et Flore/Comité d'Orientation sur la Recherche Environnementale (CAFF/CORE) statue sur le nombre ou la quantité de spécimens à collecter ou à chasser. L'autorisation de collecte ou de chasse à but scientifique n'exclut pas les autres procédures administratives nécessaires pour l'exportation. Récemment (2009), les autorisations de capture et collecte de spécimens au niveau des Aires Protégées ont été suspendues dans les aires protégées gérées par Madagascar National Park (C. Andrianarivo, comm. pers.).

L'autorisation de collecte ou de chasse à but commercial porte sur les espèces protégées (Cat. 1.2), les espèces nuisibles (Cat. 2) et les espèces de gibier (Cat. 3). Pour obtenir une autorisation de collecte ou de chasse commerciale, les opérateurs doivent disposer d'un centre de stockage agréé pour l'exportation d'animaux vivants; Ies autorisations sont assujetties à un quota annuel pour chaque espèce concernée. Toute personne désirant obtenir ce type d'autorisation doit envoyer une demande annuellement à la DGF qui, après avoir donné son accord, I'envoie à la DREF concernée par la collecte ou la capture pour que celle-ci établisse une autorisation valable pour une période d'un an. Dans le cas du commerce local, les titulaires de ce type d'autorisation doivent suivre 
les dispositions fiscales et payer les redevances auprès de I'Administration Forestière.

LE DROIT D'USAGE. Suivant I'article 3 de I'arrêté

327-MAP/FOR du 6 février 1961, les populations locales bénéficient d'un droit d'usage et peuvent ainsi prélever du gibier (Cat. 3) sans avoir à demander une autorisation à condition que la chasse se déroule pendant la période d'ouverture de la chasse et dans les zones autorisées. Ce droit d'usage n'est concédé que pour assurer des besoins personnels de la population, de sorte que le gibier ainsi obtenu ne pourra faire l'objet d'aucune transaction commerciale. En aucun cas, les animaux protégés ne peuvent être abattus au titre des droits d'usage. La chasse avec des armes de fabrication locale (notamment sagaies, arcs, sarbacanes) et le piégeage sont autorisés au titre du droit d'usage, sauf dans les régions où la détention desdites armes est interdite.

CHASSE ET SYSTĖME DES AIRES PROTÉGÉES. Les objectifs

du Système des Aires Protégées à Madagascar sont de conserver l'ensemble de la biodiversité unique de l'île (écosystèmes, espèces, variabilité génétique), de conserver le patrimoine culturel malgache, de maintenir les services écologiques et l'utilisation durable des ressources naturelles pour la réduction de la pauvreté et le développement durable. Les premières créations de parcs et de réserves remontent à la période coloniale avec la création de Réserves Naturelles Intégrales, de Parcs Nationaux, de Réserves Spéciales et de réserves de chasse. Ce système à été récemment étendu et mis à jour en mettant un plus fort accent sur I'utilisation durable. Le Code de gestion des Aires Protégées (COAP, Ioi 2001-005, décret 2005-848) classifie les aires protégées de Madagascar en quatre catégories : parc naturel, monument naturel, paysage harmonieux protégé et réserve de ressources naturelles. L'utilisation des ressources pour la subsistance est autorisée dans toutes les catégories suivant leurs plans d'aménagement respectifs. L'utilisation des ressources à des fins commerciales n'est pas autorisée ni dans les parcs, ni dans les monuments naturels, mais peut être pratiquée dans les autres catégories suivant les accords locaux, le décret 2006-400 et le plan d'aménagement. Une révision du COAP en accord avec les différentes catégories de I'UICN est en cours de préparation depuis 2008.

\section{DISCUSSION}

La conservation des ressources naturelles n'est pas une idée nouvelle à Madagascar. Bien avant I'acquisition de I'indépendance en 1960, de nombreuses aires protégées avaient été créées dans ce dessein et Madagascar fut l'un des premiers pays à établir son système des aires protégées (décret 66-242 en 1960). Cet effort se poursuit toujours et l'État malgache affiche clairement sa volonté de conserver ses ressources naturelles notamment en augmentant la superficie du réseau des aires protégées du pays qui passera de 1,7 millions d'hectare à 6 millions d'hectares sur une période de cinq ans. Si Madagascar dispose d'un cadre juridique étoffé pour la protection et la régulation de la chasse des espèces sauvages, il existe cependant quelques anomalies dans leur application par rapport à la classification de I'UICN, à la CITES, I'écologie des espèces et la réalité locale.

CATÉGORIES. Les catégories appliquées depuis

2006 constituent une bonne base pour distinguer les espèces pouvant être exploitées et les espèces protégées à Madagascar. Nous avons cependant relevé que la catégorie des espèces protégées mais pouvant aussi être exploitées (Cat. 1.2) est à l'origine d'une certaine confusion, car si elle est adaptée au commerce international, sous l'égide de la CITES, elle s'avère pesante pour les exploitations locales. Par exemple, le tenrec Setifer setosus et la grenouille allochtone Hoplobatrachus tigerinus qui sont des espèces prisées pour la consommation locale, sont vendues directement sur les marchés locaux ou dans les restaurants. Comme elles sont des espèces protégées (Cat. 1.2) et inscrites à l'annexe 2 de la CITES, leur exploitation nécessite une autorisation émanant de la DGF (Antananarivo) et elles sont soumises à un quota chaque année. De plus, il existe un cahier des charges auquel l'exploitant doit se soumettre. Les démarches à suivre pour l'obtention de ce type d'autorisation sont difficiles voire impossibles à réaliser pour les chasseurs des milieux ruraux, de sorte que cette loi n'est pas appliquée. De même, il semble anormal que des espèces comme les chauvessouris frugivores qui sont menacées d'extinction par la chasse soient dans la catégorie du gibier en pouvant être chassées sans qu'aucune mesure de conservation ne puisse être prise pendant la saison d'ouverture de la chasse.

L'efficacité de la législation existante dépend étroitement de son application. Plusieurs éléments montrent que les autorités déploient peu d'efforts pour sensibiliser le grand public à la loi sur les espèces protégées ou à la faire appliquer. Par exemple, il n est pas rare de noter la détention en captivité de lémuriens ou de tortues dans les ménages à Madagascar alors qu'il est illégal de tuer, de vendre ou de garder ces animaux en captivité sans une autorisation appropriée.

LISTE ROUGE DE L'UICN. La Liste Rouge de I'UICN est

un outil indispensable d'aide à la prise de décision de l'État dans la gestion et la protection de la diversité biologique. Puisque les statuts de conservation des espèces doivent être mis à jour au moins tous les dix ans, la Liste Rouge de I'UICN est relativement dynamique, est la mieux placée pour intégrer les avancements dans la taxinomie et les changements dans la distribution ou l'abondance des espèces. Les espèces menacées d'extinction sont par conséquent celles ayant le plus besoin de mesures de conservation. Ainsi, on peut penser que toutes les espèces qui figuraient dans la catégorie En Danger Critique d'Extinction en 2005 auraient du bénéficier d'une protection absolue (Cat. 1.1) dans le décret adopté en 2006. De fait, la majorité de ces espèces sont intégralement protégées dans ce décret mais trois espèces ont été maintenues dans la Cat. 1.2 car elles étaient proposées dans le commerce international avant 2006 et que cette option a voulu être perpétrée. Un grand nombre d'espèces qui ne sont pas menacées d'extinction bénéficient d'une protection absolue dans la législation malgache ; ceci est par exemple le cas avec l'inclusion de toutes les espèces de lémuriens dans la Cat. 1.1 sans distinction de statut.

CITES. La possibilité d'ajouter ou de supprimer des espèces inscrites aux différentes annexes de cette convention est limitée car tout changement doit être agréé au niveau de la Conférence des Parties qui n'a lieu que tous les quatre ans. II apparaît d'ailleurs que la révision de la loi portant sur la faune sauvage en 2006 s'est alignée du mieux qu'elle pouvait sur la liste des espèces inscrites aux annexes de la CITES. Les espèces inscrites à l'annexe 1 sont ainsi strictement protégées et celles à l'annexe 2 bénéficient d'une certaine protection mais peuvent être collectées ou chassées sur autorisation de l'État. Cette approche paraît raisonnable, à quelques exceptions comme dans le cas de Setifer setosus qui se trouve 
dans la Cat. 1.2, un cas difficile à expliquer dans la mesure où il s'agit d'une espèce commune et consommée par la population.

AUTRES CONVENTIONS. Madagascar se doit de protéger légalement certaines espèces conformément aux conventions qu'elle a signées, et interdire ou contrôler la chasse des espèces mentionnées dans lesdites conventions. Les espèces concernées et qui peuvent prétendre à un certain niveau de protection sont la Baleine Bleue Balaenoptera musculus, la Baleine à Bosse Megaptera novaeangliae et le dugong Dugong dugon alors qu'aucune de ces trois espèces ne figurent dans la liste des espèces protégées de Madagascar établie en 2006.

LA PÊCHE. Les lois formulées par le MEF et le MPRH devraient être complémentaires en matière de protection et d'utilisation durable des espèces, mais on remarque qu'en l'état, elles semblent être une source de confusions. Par exemple, les mammifères marins (cas des baleines, des dauphins et du dugong) sont protégés conformément à une loi sur la pêche maritime mais ne sont pas inclus dans la liste la plus récente des espèces animales protégées établie par le MEF, alors que les tortues marines figurent dans les deux lois. De même, la gestion des amphibiens comestibles (Hoplobatrachus tigerinus, Mantidactylus grandidieri et Boophis goudoti) est actuellement attribuée au MPRH alors que ces amphibiens sont inscrits dans la loi du MEF. La gestion de ces trois espèces d'amphibiens est tiraillée entre deux législations ; dans la réalité, le niveau de collecte augmente mais ne peut être réglementé (Jenkins et al. 2009).

CHASSE COMMERCIALE ET DROIT D'USAGE. La chasse est

qualifiée de commerciale lorsque les produits sont vendus sur le marché, que ce soit au niveau local, régional, national ou international ; le chasseur devrait alors détenir une autorisation de chasse appropriée conformément à I'ordonnance 1960-126). À Madagascar, la population rurale utilise des produits de chasse comme source de revenus afin de subvenir aux besoins de subsistance, ce qui va à l'encontre du droit d'usage. L'absence de détention d'une autorisation par ces chasseurs locaux peut être expliquée en partie par la lourdeur des formalités administratives inhérentes à la procédure. En effet, I'autorisation est émise par la DGF ou la DREF, de sorte que les coûts de déplacement peuvent parfois être plus élevés que les bénéfices issus de la vente des produits de la chasse.

SAISONS ET CONDITIONS DE CHASSE. Certains passages des lois et règlements portant sur la faune sauvage à Madagascar paraissent difficiles à adapter aux réalités locales, dont des aspects relatifs à la commercialisation des produits, la saison d'ouverture de la chasse et les conditions dans lesquelles elle peut être pratiquée pour certaines espèces. Les saisons d'ouverture et de fermeture de la chasse sont définies annuellement pour le gibier et doivent être respectées. Or, les chasseurs ciblent les espèces suivant leur mode de vie, en favorisant par exemple les périodes au cours desquelles les animaux sont le plus facile à capturer où lorsqu'ils sont les plus gras. Ainsi, certaines espèces comme Tenrec ecaudatus sont chassées en dehors de la saison définie par la loi. De même, la chasse ne peut être pratiquée de nuit selon la loi, mais dans la liste des gibiers figurent des espèces nocturnes comme les chauves-souris. Pour les chasseurs, il est plus commode de capturer ces chauves-souris lorsqu'elles viennent s'alimenter la nuit près des villages, et c'est ce qui est souvent pratiqué. Si ces animaux devaient être chassés au cours de la journée, les chasseurs seraient amenés à perturber les gîtes et causer un dérangement néfaste. Les conditions pratiques et la biologie des espèces devraient donc être considérées avant la définition des saisons d'ouverture et de fermeture de la chasse afin de légaliser la chasse de subsistance locale.

\section{RECOMMANDATIONS}

À l'issue de cette revue, huit dispositions sont proposées:

1. À partir des catégories des espèces protégées figurant dans le décret 2006-400, en tenant compte de toutes les lois et réglementations existantes, établir une nouvelle liste complète de tous les animaux qui reflèterait les statuts de conservation et les importances en matière de subsistance pour la population rurale. Les procédures en vue de permettre l'actualisation régulière de cette liste devrait aussi être mises en place ;

2. Réviser les saisons d'ouverture et de fermeture de la chasse de façon à mieux refléter la réalité, en tenant compte de la biologie des espèces ainsi que des considérations pratiques pour la chasse et la détention des animaux ;

3. Renforcer l'application de la loi, plus particulièrement I'interdiction de chasser les espèces protégées, le contrôle de I'utilisation des fusils et la commercialisation des animaux chassés dans le cadre du droit d'usage ;

4. Faire en sorte que les lois et règlements sur la faune et la biodiversité en général soient plus facilement consultables par tous les acteurs, parmi lesquels les personnels du MEF, du MPRH, du Ministère de I'Intérieur, les différentes organisations de conservation et de développement ;

5. Fournir aux personnels actifs sur le terrain (ex. les agents des ministères, les gendarmes) des matériels et formations adaptés portant sur la législation et I'environnement ;

6. Faciliter les procédures d'obtention des autorisations de chasse, particulièrement pour la chasse à but commercial afin de régulariser les ventes du gibier sur les marchés locaux;

7. Publier tous les textes sur l'exploitation et la protection des espèces sauvages sur les sites web des ministères concernés au niveau du gouvernement ;

8. Conformément aux décisions prises lors de la Conférence des Parties de la CDB, chaque pays membre, dont Madagascar, doit adopter des mesures afin de réduire la chasse illégale et non durable et promouvoir la chasse durable. Ainsi, il est recommandé que I'exploitation des animaux sauvages soit considérée dans les futures stratégies nationales de conservation de la biodiversité.

\section{CONCLUSION}

Madagascar dispose d'un cadre juridique pour la protection et la régulation de la chasse des espèces de la faune sauvage. Cependant, afin que les lois et règlements au niveau national soient en harmonie avec les réalités locales, les conventions internationales et l'avis des scientifiques, une révision de la liste sur les annexes du décret 2006-400 est nécessaire. L'application 
de la loi pourrait être effective si les procédures d'obtention des autorisations de chasse étaient simplifiées et que les personnes impliquées directement sur le terrain disposent des connaissances et des outils nécessaires.

\section{REMERCIEMENTS}

Nous tenons à présenter toute notre gratitude aux responsables du Ministère de l'Environnement et des Forêts pour avoir autorisé la conduite de cette revue dans le cadre du Programme Gestion Durable de la Biodiversité, Éducation et Sensibilisation Environnementale de Madagasikara Voakajy, en collaboration avec I'Université de Bangor et du Département de Biologie Animale de I'Université d'Antananarivo. Nous sommes reconnaissants aux nombreuses personnes qui nous ont aidé lors de la documentation, ce qui nous a permis de rassembler un grand nombre de textes et lois portant sur la chasse à Madagascar, notamment les personnels des trois directions auprès du Ministère de l'Environnement et des Forêts (MEF) au sein de la Direction Générale des Forêts (DGF) sises à Antananarivo, la Direction de la Conservation de la Biodiversité et du Système des Aires Protégées (DCBSAP), la Direction de la Valorisation des Ressources Naturelles (DVRN) et la Direction Régionale de I'Environnement et des Forêts (DREF) Analamanga. Nos sincères remerciements s'adressent particulièrement à Andriantahiry Rabesandratana et Andrew Cooke pour leur aide précieuse ; à Lydie Raharimaniraka (DVRN) et Barbara Livoreil (Université de Bangor) qui ont bien voulu commenter ce manuscrit avant sa soumission, et aux trois rapporteurs anonymes qui ont apporté leurs suggestions et commentaires en améliorant sensiblement ce travail. Nous sommes reconnaissants à Darwin Initiative pour nous avoir fourni les ressources financières nécessaires pour effectuer cette revue.

\section{RÉFÉRENCES}

Andriantompohavana, R., Lei, R., Zaonarivelo, J. R. Engberg, S. E., Nalanirina, G, McGuire, S. M., Shore, G. D., Andrianasolo, J., Herrington, K., Brenneman, R. A \& Louis Jr., E. E. 2007. Molecular phylogeny and taxonomic revision of the woolly lemurs, genus Avahi (Primates: Lemuriformes). Special Publication. Museum of Texas Tech University. Number 51: 1-59.

Bakarr, I. M., de Fonseca, G. A. B., Mittermeier, R., Rylands, A. B. \& Painemilla, K. W. 2001. Hunting and Bushmeat Utilization in the African Rainforest. Advances in Applied Biodiversity Science. Number 2. Centre for Applied Biodiversity Science, Conservation International, Washington, D.C.

Barrett, M. A. \& Ratsimbazafy, J. 2009. Luxury bushmeat trade threatens lemur conservation. Nature 461: 470. <doi:10.1038/461470a>

Cooke, A. J. (sous presse). Madagascar - A Guide to Marine Biodiversity. Wildlife Conservation Society. Editions Resolve, Antananarivo.

Corlett, R. T. 2007. The impact of hunting on the mammalian fauna of tropical Asian forests. Biotropica 39, 3: 292-303. <doi:10.111 1/j.1744-7429.2007.00271.x>

Craul, M., Zimmermann, E., Rasoloharijaona, S., Randrianambinina, B. \& Radespiel, U. 2007. Unexpected species diversity of Malagasy primates (Lepilemur spp.) in the same biogeographical zone: A morphological and molecular approach with the description of two new species. BMC Evolutionary Biology 7, 83: 1-15. <doi:10.1186/14712148-7-83>

Draper, R. 2010. Madagascar's Pierced Heart. National Geographic Magazine 218: 80-109.

Fa, J. E., Peres, C. A. \& Meeuwig, J. 2002. Bushmeat exploitation in tropical forests: An intercontinental comparison. Conservation Biology 16, 1 232-237. <doi:10.1046/j.1523-1739.2002.00275.x>
García, G. \& Goodman, S. M. 2003. Hunting of protected animals in the Parc National d'Ankarafantsika, north-western Madagascar. Oryx 37, 1: 115-118. <doi:10.1017/S0030605303000206>

Glaw, F. \& Vences, M. 2007. A Fieldguide to the Amphibians and Reptiles of Madagascar. Third Edition. Vences \& Glaw Verlag. Cologne.

Golden, C. D. 2009. Bushmeat hunting and use in the Makira Forest, northeastern Madagascar: A conservation and livelihoods issue. Oryx 43, 3 386-392. <doi:10.1017/S0030605309000131>

Goodman, S. M. \& Benstead, J. P. 2005. Updated estimates of biotic diversity and endemism on Madagascar. Oryx 39, 1: 73-77. <doi:10.1017/ S0030605305000128>

Goodman, S. M. \& Raselimanana, A. P. 2003. Hunting of wild animals by Sakalava of the Menabe region: A field report from Kirindy-Mite. Lemur News 8: 4-6.

Goodman, S. M., Soarimalala, V. \& Ganzhorn, J. U. 2004. La chasse aux animaux sauvages dans la forêt de Mikea. In: Inventaire Floristique et Faunistique de la Forêt de Mikea: Paysage Écologique et Diversité Biologique d'une Préoccupation Majeure pour la Conservation. A. P. Raselimanana \& S. M. Goodman (eds.), pp 95-100. Recherches pour le Développement. Série Sciences Biologiques. Centre d'Information et de Documentation Scientifique et Technique 21, Antananarivo.

Goodman, S. M., Maminirina, C. P., Bradman, H. M., Christidis, L. \& Appleton, B. 2009. The use of molecular phylogenetic and morphological tools to identify cryptic and paraphyletic species: Examples from the diminutive long-fingered bats (Chiroptera: Miniopteridae: Miniopterus) on Madagascar. American Museum Novitates 3669: 1-34. <doi:10.1206/652.1>

Jenkins, R. K. B. \& Racey, P. A. 2008. Bats as bushmeat in Madagascar. Madagascar Conservation and Development 3, 1: 22-30.

Jenkins, R. K. B., Rabearivelo, A., Chan, C. T., Andre, W. M., Randrianavelona, R. \& Randrianantoandro, J. C. 2009. The harvest of endemic amphibians for food in eastern Madagascar. Tropical Conservation Science 2, 1: 25-33.

Keane, A., Jones, J. P. G., Edwards-Jones, G. \& Milner-Gulland, E. J. 2008. The sleeping policeman: Understanding issues of enforcement and compliance in conservation. Animal Conservation 11, 2: 75-82. <doi:10.111 1/j.1469-1795.2008.00170.x>

Kiszka, J., Muir, C., Poonian, C., Cox, T. M., Amir, O. A., Bourjea, J., Razafindrakoto, Y., Wambiji, N. \& Bristol, N. 2008. Marine mammal bycatch in the Southwest Indian Ocean: Review and need for a comprehensive status assessment. Western Indian Ocean Journal of Marine Science 7, 2:119-136.

Myers, N., R. A. Mittermeier, R. A., Mittermeier, C. G., da Fonseca, G. A. B. \& Kent, J. 2000. Biodiversity hotspots for conservation priorities. Nature 403: 853-858. <doi:10.1038/35002501>

Nicoll, M. E. 2003. Tenrec ecaudatus, Tenrec. In: The Natural History of Madagascar. S. M. Goodman and J. P. Benstead (eds.), pp 1423-1432. The University of Chicago Press, Chicago.

Peres, C. A. \& Terborgh, J. W. 1995. Amazonian nature reserves: An analysis of the defensibility status of existing conservation units and design criteria for the future. Conservation Biology 9, 1: 34-46. < doi:10.104 6/j.1523-1739.1995.09010034.X>

Perez, V. R., Godfrey, L. R., Nowak-Kemp, M., Burney, D. A., Ratsimbazafy, J. \& Vasey, N. 2005. Evidence of early butchery of giant lemurs in Madagascar. Journal of Human Evolution 49, 6: 722-742. <doi:10.1016/j.jhevol.2005.08.004>

Rakotondravony, H. 2006. Communautés locales et gibiers dans la région de Daraina, extrême Nord-Est de Madagascar. Madagascar Conservation \& Development 1: 19-21.

Rakotonirina, B. \& Cooke, A. J. 1994. Sea turtles of Madagascar: Their status, exploitation and conservation. Oryx 28: 51-61. <doi:10.1017/ S0030605300028295>

Randriamanalina, M. H., Rafararano, L., Babary, L. et Laha, R. 2000. Rapport des enquêtes sur les chasses dans les Fokontany d'Ivondro, d'Erara et d'Etsilesy. Lemur News 5: 11-14.

Randrianandrianina, F. H., Racey, P. A. \& Jenkins, R. K. B. 2010. Hunting and consumption of mammals and birds by people in urban areas of western Madagascar. Oryx 44, 3: 411-415. <doi:10.1017/ S003060531000044X> 
Wilkie, D. S., Sidle, J. G. \& Boundzanga, G. C. 1992. Mechanized logging, market hunting, and a bank loan in Congo. Conservation Biology 6, 4 570-580. <doi:10.1046/j.1523-1739.1992.06040570.x>

Wilkie, D. S., Carpenter, J. F. \& Zhang, Q. F. 2001. The under financing of protected areas in the Congo Basin: so many parks and so little willingness to pay. Biodiversity and Conservation 10, 5: 691-709.

MATÉRIEL SUPPLÉMENTAIRE.

DISPONIBLE EN LIGNE.

Tableau S1. Niveau de protection des espèces protégées suivant les conventions internationales et la législation nationale mais sans protection selon le décret 2006-400.

Tableau S2. Les espèces classées en tant que gibier dans la législation malgache et qui sont menacées (ou quasi-menacées) d'après les critères de I'UICN.

Tableau S3. Lois et règlements portant sur la gestion des espèces de la faune sauvage.

Tableau S4. Ensemble des 1137 espèces considérées dans I'analyse des lois et règlements portant sur la faune sauvage à Madagascar. 